\title{
Evaluating on Topology Survivability Based on Largest Number of Node-Disjoint Paths
}

\section{Qiurong Chen*}

Shijiazhuang No. 24 High School, No. 224 Ziqiang Road, Shijiazhuang City 050002, Hebei Province, China. Email: 1989601713@qq.com

Abstract: On the point of view of Largest Number of Node-Disjoint Path (LNNDP for short) between a node pair in a network, this article states the importance of LNNDP to global survivability of topology at first, then proposes an algorithm to compute maximal number of node-disjoint paths between node pairs. A new topology survivability metric based on LNNDP is put forward to evaluate the global survivability of network topology. It can be used to evaluate the survivability of topology provided. This metric can express accurately global topology survivability.

Keywords: Topology; Survivability; Metric; Node-Disjoint Path; Working Path; Alternate Path

\section{Introduction}

Topology survivability is the basis of the survivability in multi-layered networks. It is well known that adopting mesh type topology with link redundancy is more cost effective and flexible way which can keep network connected when failures of network elements (nodes or links) occur. Topology survivability is much dependent on the redundant links' placements in a network, because the placement variations are closely related to the number of node-disjoint path (NNDP for short) between any node pair. This article focuses on node-disjoint paths rather than link-disjoint paths [1-3], because node-disjoint paths must be of link-disjoint, it is very useful for fault-tolerance in topology, and is very significant in wireless mobile networks. The node-disjoint path between node pair plays very important role to survivable topology, because it is very necessary for working path between node pair switching to an alternate node-disjoint path immediately, so as to guarantee topology survivability and path availability when limited links or nodes fail randomly in a network. In addition, node-disjoint path can be usually used to share traffic load, to improve throughput, and to balance burst traffic. The authors focus on the effectiveness of node-disjoint path on topology survivability of network in this paper.

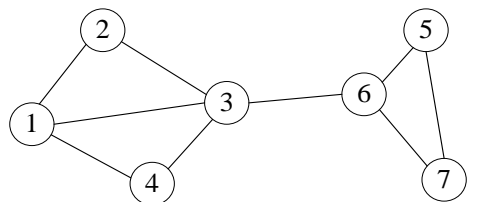

Figure 1. Functions of node-disjoint path.

As shown in Figure 1, there is only one node-disjoint path for node pair $(1,7)$ at same time. This node pair must be disconnected while node 3 or node 6 or link 3-6 fails, because node 3 or 6 is joint node, and link 3-6 is joint link for all paths between this node pair. Node pair $(1,3)$ is fortunate, it has 3 node-disjoint paths, namely path $1-3$, path $1-2-3$, and path 1-4-3. When any two elements between the two nodes fail, this node pair

Copyright (C) 2020 Qiurong Chen

doi: 10.18282/jnt.v2i3.1107

This is an open-access article distributed under the terms of the Creative Commons Attribution Non-Commercial License

(http://creativecommons.org/licenses/by-nc/4.0/), which permits unrestricted non-commercial use, distribution, and reproduction in any medium,

provided the original work is properly cited. 
will still be connected. This survivable capability for this node pair benefits from the three node-disjoint paths.

It is obvious that largest number of node-disjoint path (LNNDP for short) exists between any node pair, for example, the LNNDP of node pair $(1,3)$ is 3 . The LNNDP of a node pair is very important to topology survivability, because the larger the LNNDP of a node pair is, the better the survivable capacity of this node pair is. Some references showed that computing Maximal Set of Node-Disjoint Path (MSNDP for short) or LNNDP between any node pair in a network is possible. A route protocol, which is called Node-Disjoint Multipath Routing Protocol (NDMR), was proposed by Li and Cuthbert $^{[4]}$, and it can discover multiple node-disjoint routing paths, but not for MSNDP. A hybrid protocol for identification of a MSNDP in mobile Ad Hoc networks was proposed by $\mathrm{Ash}^{[5]}$, which says one can guarantee to identify a MSNDP in an incremental mode by using multiple route discoveries. A computation method of multiple paths based on node-disjoint between a pair of source and destination was put forward by Nagaratna et al. ${ }^{[6]} \mathrm{A}$ paper $^{[7]}$ presented a new algorithm to find the largest number of node-disjoint paths which exist at the same time in the network. The problem of constructing the maximal number of node-disjoint paths between two distinct nodes in Swapped/OTIS networks was investigated by Chen et al. ${ }^{[8]}$

One node pair may have one or more MSNDPs, and LNNDP must exist in some a MSNDP. For example, in the topology shown in Figure 2, node pair (1,7) has three maximal sets of node-disjoint path, which are \{1-3-6-7, $1-4-7\},\{1-3-6-5-7,1-4-7\}$, and $\{1-2-3-6-7,1-4-7\}$, and the LNNDP is two.

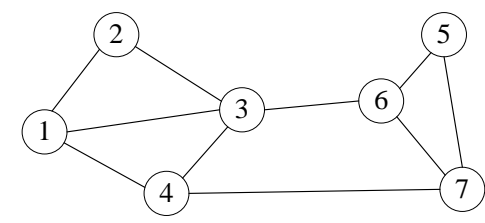

Figure 2. LNNDP from MSNDP.

The LNNDPs between node pairs are different from each other at most cases because of the variations of redundant links' placement in a network. If the LNNDP between a node pair is $k(k \geq 2)$, then this node pair will still be connected when $(k-1)$ network elements (nodes, or links, or nodes plus links) fail. Unfor- tunately, we do not know which elements will fail while the network is operating, because it is unpredicted. So to guarantee any node pair to be connected in topology when any $(k-1)$ network elements fail, the LNNDP between any node pair should be at least $k$. Particularly if the LNNDP of any node pair is of the same value $k$, then any $(k-1)$ elements' failure has the same influence on working path switching to alternate path. In another word, any node pair has the same survivable performance. This is the reason that the most simple and survivable optical network topology is of ring type which has the same LNNDP value (i.e. two) for any node pair. This criterion is in accord with that described $\mathrm{in}^{[9]}$, it says that the most survivable network topologies, are those in which no node appears to be more critical than any other node and no link appears to be more critical than any other link.

The topology survivability requirements of communication network must be satisfied when it is designed. $\mathrm{In}^{[10]}$, it described that survivability requirements refer to system capabilities for the delivery of essential services in the presence of attacks and intrusions, and recovery of full services. Topology survivability is the focus of this article. So the survivability of topology should aim to the capability of keeping any node pair connected when any one or more elements fail at the same time in presence of various attacks. This criterion is much related to the LNNDP of all node pairs in a network.

The survivable topology can guarantee that any node pair will still be connected when any $(k-1)$ elements fail if any node pair's LNNDP is at least $k$. Usually the LNNDP of node pair may be different from each other, maximal and minimal LNNDPs must exist among them. $L N N D P_{\max }$ denotes maximal LNNDP, and $L N N D P_{\min }$ minimal LNNDP. $L N N D P_{\text {min }}$ is very significant for global topology survivability, which means that any node pair will be connected when any $\left(L N N D P_{\text {min }}-1\right)$ elements fail in the network. The difference between $L N N D P_{n p}$ and $L N N D P_{\text {min }}$ is also beneficial for the topology survivability. In particular, any node pair has the same value, it means LNNDP is equal to $L N N D P_{\max }$, and equal to $L N N D P_{\min }$. Hence the authors put forward a new topology survivability metric $T S_{\text {lnndp }}$ which is based on NDP's diversity in a network. This metric can be used to evaluate topology survivability designed. The rest of the article is organized as fol- 
lowing. Section 2 describes some researches in related areas. Section 3 states the problem's mathematical model. Section 4 gives the metric formulation. In Section 5, an algorithm is proposed. In Section 6, numeric results are given based on the algorithm proposed. Section 7 provides applications of the proposed topology survivability metric, and Section 8 concludes the article.

\section{Related works}

Several topological survivability metrics were reviewed in ${ }^{[11]}$, they are "Node Degree”, “Total Graph Diversity (TGD for short)", “Clustering Coefficient”, "Diameter”, "Radius”, “Hop-Count”, “Closeness”, and "Betweenness", and a new topology survivability metric cTGD (Compensated Total Graph Diversity) was created based on TGD. It was stated that CTGD was an excellent predictor of the survivability of topologies than others reviewed above.

Other commonly-used topology survivability metrics are MinCut (the minimal number of links whose deletion can disconnect the node pair) and MaxFlow (the maximal number of link-disjoint paths between the node pair) stated $\mathrm{in}^{[12]}$. If MinCut or MaxFlow equals to $k$, the node pair is still connected when any $k-1$ link failures (Menger's Theorem). MinCut and MaxFlow can be computed efficiently (Ford-Fulkerson Algorithm).

$\mathrm{In}^{[13]}$, an algebraic connectivity, adopted from spectral graph theory, namely the second smallest Eigenvalue of the Laplacian Matrix of the network topology, was proposed, which can characterize a network's survivability of topology better than the Average Node Degree (AND for short) that has been traditionally used to suggest fault-tolerance capability of network topology.

Different from the metrics mentioned above, a new topology survivability of network is put forward in this article from the point of view of LNNDP of node pair.

\section{Math model}

\subsection{LNNDP between node pair}

As described in Section 1, to guarantee any node pair to be connected in topology when any $k-1$ network elements fail, the LNNDP between any node pair should be at least $k$. The larger the $L N N D P_{\min }$ is, the better the network topology survivability will be.

The LNNDP between any node pair should be at least two to achieve the least survivability. $\mathrm{N}$ denotes node amount in a topology, and the LNNDP for any node pair must be smaller or equal to $(\mathrm{N}-1)$.

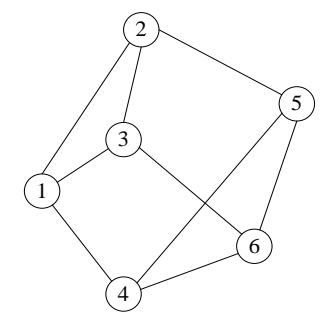

Figure 3. Topology (7 nodes 12 links).

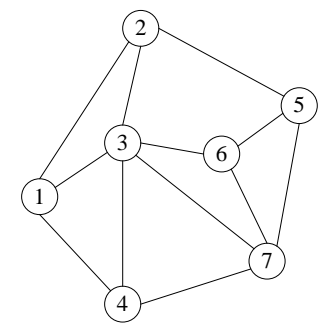

Figure 4. Topology (6 nodes 9 links).

For example, shown as Figure 3, the minimal LNNDP is 3, and maximal LNNDP is 4. Any node pair will have alternate path available when any two elements (2 nodes, 2 links, or 1 node plus 1 link) fail.

\subsection{Case of equal LNNDP}

When LNNDP of any node pair in a topology is the same, every node pair in the network will have the same working path restoration capacity. There is no node pair appearing to be more critical than any other node pair in this case. It is the most survivable topology on the global view.

As shown in Figure 4, any node pair in the topology has the same LNNDP. Any node pair can switch exactly the working path to the alternate path when any two elements fail. Taking node pair $(1,6)$ as an example, if the working path is 1-4-6, when node 3 and 4 fail simultaneously, the path 1-2-5-6 will become a new working path.

\subsection{Case of different LNNDP}

The LNNDP of node pair may be different from one another at some cases. $L N N D P_{n p}$ denotes any node pair' LNNDP, and $L N N D P_{n p}$ is divided into two parts. One part is $L N N D P_{\min }$, another is $\left(L N N D P_{n p}-\right.$ $\left.L N N D P_{\min }\right)$. Part one exactly guarantees that any node pair will have alternate path available when any two elements in a network fail. Part two will improve global topology survivability in fraction. Taking the topology 
shown as Figure 3 as an example, the LNNDP of node pair $(3,7)$ is 4 , and LNNDPs of other node pairs are 3. Three of the LNNDP of node pair $(3,7)$ together with the LNNDPs of other node pairs make any node pair to have exactly three LNNDPs. The remainder of the LNNDP of node pair $(3,7)$ will still function to global survivability of topology.

\section{Metric formulating}

\subsection{LNNDPs of node pair}

For a network with the node amount $N(N>3)$, NP denotes the number of node pair.

$N P=\frac{N(N-1)}{2}$

\subsection{Functions of LNNDP}

For a survivable topology designed, while it works, one or more elements may fail because of various attacks. LNNDP $_{\text {min }}$ will be available for any node pair, it functions to ensure that any node pair will has alternate path available when $\left(L N N D P_{\min }-1\right)$ elements fail. If more than $\left(L N N D P_{\text {min }}-1\right)$ elements fail at a time, not all node pair has alternate path available. It is necessary to sum the alternate paths beyond the $L N N D P_{\min }$ (i.e. $L N N D P_{n p}-L N N D P_{\text {min }}$ ) for each node pair, because this summation plays an incomplete role to global topology survivability. This summation's proportion to total amount of LNNDPs in the topology should also be paid attention to.

\subsection{Topology survivability metric}

All LNNDPs' functions should be taken into account when failures of elements happen. So $\mathrm{TS}_{\text {Inndp }}$ is defined as follows:

$T S_{\text {lnndp }}$

$=L N N D P_{\text {min }}+\frac{\sum_{n p=1}^{N P}\left(L N N D P_{n p}-L N N D P_{\text {min }}\right)}{\sum_{n p=1}^{N P} L N N D P_{n p}}$

Formula (3) comprises of two parts. The first part is $L N N D P_{\text {min }}$, which determines mainly the global survivability of topology. The second part is the complementary fraction.

According to Formula (3), the minimal value of $T S_{\text {lnndp }}$ is $L N N D P_{\text {min }}$. It is the integral part of the metric. $T S_{\min }$ denotes the minimal value of the metric.

Formula (3) can be transformed into Formula (4).

$T S_{\text {lnndp }}=L N N D P_{\min }+1-\frac{\sum_{n p=1}^{N P} L N N D P_{\min }}{\sum_{n p=1}^{N P} L N N D P_{n p}}$
According to Formula (4), the maximal value of $T S_{\text {lnndp }}$ ( $T S_{\max }$ denotes it) is represented as Formula (5).

$$
\begin{aligned}
T S_{\text {lnndp }}(\max )= & L N N D P_{\min }+1 \\
& -\frac{N P \cdot L N N D P_{\min }}{N P \cdot L N N D P_{\max }}
\end{aligned}
$$

Formula (5) can be simplified to Formula (6).

$T S_{\text {lnndp }}(\max )$

$=L N N D P_{\text {min }}+\frac{L N N D P_{\text {max }}-L N N D P_{\text {min }}}{L N N D P_{\text {max }}}$

By Formula (6), an in-equation is obtained as following.

$T S_{\max }<L N N D P_{\min }+1$

\section{Algorithm to compute LNNDP}

As described in Section 1, it is possible to compute MSNDP for a given network. Compared to computing MSNDP, it is not very hard to compute LNNDP. Note that LNNDP is just a largest number of node-disjoint path, it is different from MSNDP which contains much more information than LNNDP. An algorithm proposed here to compute LNNDP of one node pair is shown as Figure 5. This algorithm comprises of two parts which are Algorithm Part A and Algorithm Part B.

The topology survivability metric $\mathrm{TS}_{\text {lnndp }}$ can be computed as Formula (4) once all LNNDPs have been calculated.

Algorithm Part A

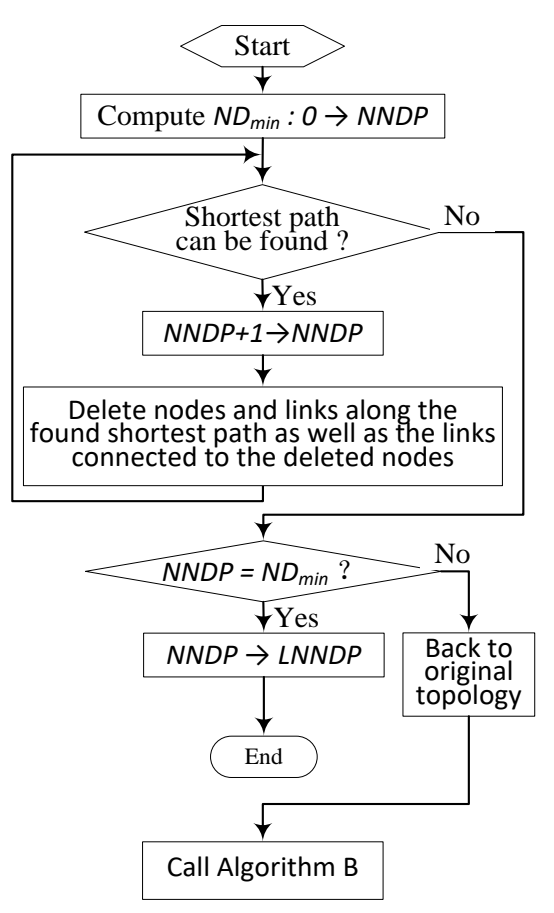




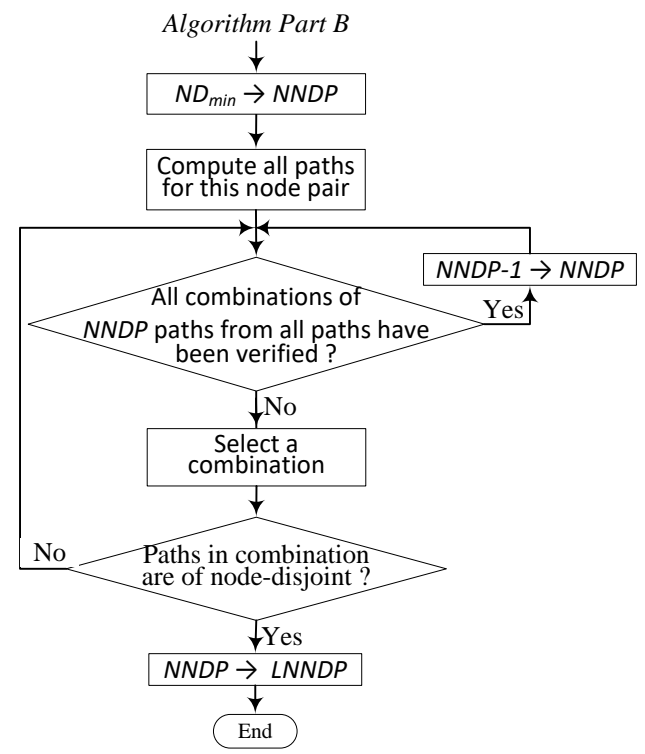

Figure 5. Algorithm to compute LNNDP of one node pair.

\section{Numeric Results}

As algorithm described above, take topology shown as Figure $\mathbf{6}$ as an example. The numeric results computed are:

$L N N D P_{\min }=2, L N N D P_{\max }=3$, and $T S_{\text {lnndp }}=$ 2.28 .

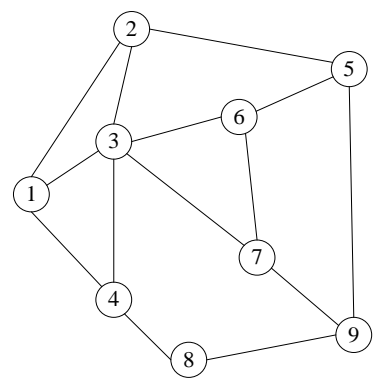

Figure 6. Topology (9 nodes 14 links).

Another example is network topology G6 shown as

Figure 7, computed results are: $L N N D P_{\min }=2$, $L N N D P_{\max }=5$, and $T S_{\text {lnndp }}=2.33$.

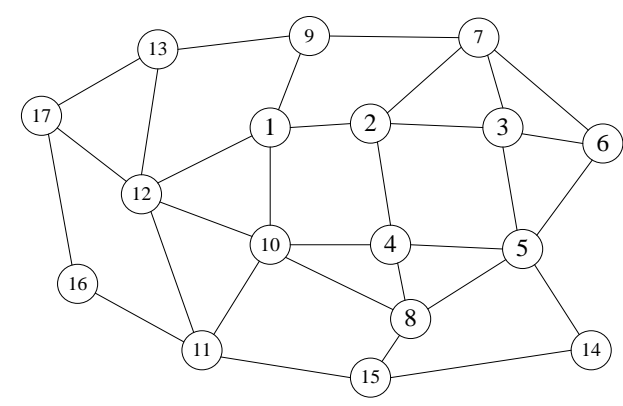

Figure 7. Topology (17 nodes 31 links).
The metric $T S_{\text {lnndp }}$ can evaluate global survivability of network's topology, and may be used in the phase of design or reconfiguration for topology.

\subsection{Applied in survivable topology design}

Network topology separation may be caused by element failures. Topology survivability may be defined in general as that any node pair will still be connected in topology when some elements fail. The failed elements may be nodes or links in a network. Therefore, the amount of randomly and simultaneously fault elements that one network topology can endure is critical. When a network topology is designed as requirements, it is usually necessary to evaluate its survivability of topology. The metric $\mathrm{TS}_{\text {Inndp }}$ proposed in this article can be adopted to evaluate, so as to predict the amount of randomly and simultaneously fault elements that the topology can tolerance. The metric $T S_{\text {lnndp }}$ of designed topologies shown as Figure 3 and Figure 4, are 3.0156 and 3.0 respectively. The topology survivability shown as Figure $\mathbf{3}$ is a little better than that shown as Figure 4, and they are almost the same.

\subsection{Applied in survivable topology adjust- ment}

After several elements in a network have been of malfunction, the survivability is degraded, so the topology should be adjusted (or reconfigured) at this time to improve its survivability if there are no more elements available. At this scenario, we may compute the metric $\mathrm{TS}_{\text {Inndp }}$ of reconfigured topology to evaluate its survivability. Taking the topology shown as Figure $\mathbf{3}$ as an example, after node 3 and link (6-7) are destroyed simultaneously, the remained topology will be shown as Figure 8, and it has no capability of global survivability, or its $\mathrm{TS}_{\text {Inndp }}$ is less than 2 (1.3488). So the designer should adjust the existing links' placement to improve the topology. The adjusted topology may be that shown as Figure 8a, 8b, 8c, and 8d, whose $T S_{l n n d p} \mathrm{~s}$ are1.1764, $1.1764,1.4285$, and 2.0. The best one among the adjusted topologies is obviously shown as Figure 8d, whose survivability metric $T S_{l n n d p}$ is exactly improved to 2 .

\section{Metric applications}




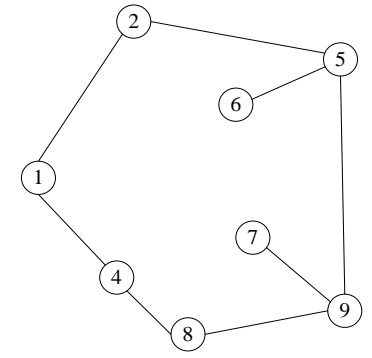

Figure 8. Topology destroyed (8 nodes 8 links remained).

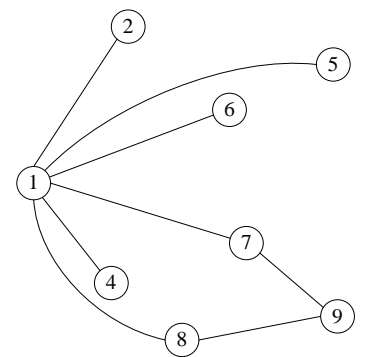

Figure 8a. Topology adjusted (star type).

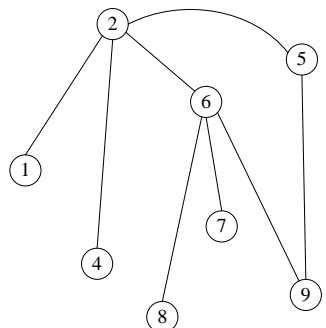

Figure 8b. Topology adjusted (tree type).

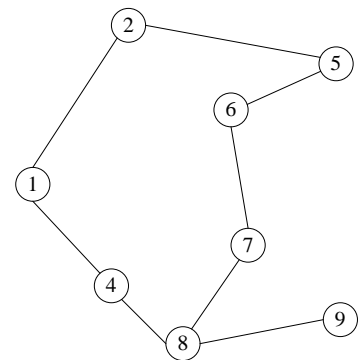

Figure 8c. Topology adjusted (near ring type).

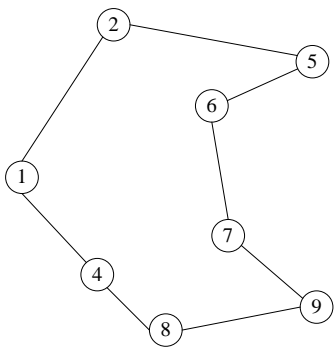

Figure 8d. Topology adjusted (ring type).

\section{Conclusion}

This article describes the importance of LNNDP to global survivability of topology, and proposes an algorithm to compute maximal number of node-disjoint paths between node pairs. The article's contribution is that it puts forward a new topology survivability metric $T S_{\text {lnndp }}$ which is based on LNNDP to evaluate the global survivability of designed topology, and upper as well as lower bounds are given. Numeric results computed are provided by examples. Applications of proposed survivability metric for topology design and adjustment are described too. This metric can express more informative global topology survivability than Average Node Degree (See Appendix).

\section{Acknowledgements}

The author would like to thank guidance of his parents and teachers in school for their invaluable contributions. Meanwhile he appreciates the anonymous referees for their careful reading as well as many good comments.

\section{References}

1. Whalen JS, Kenney J. Finding maximal link disjoint paths in a multigraph. IEEE Global Telecommunications Conference IEEE; 1990.

2. Loh RC, Soh S, Lazarescu M. An approach to find maximal disjoint paths with reliability and delay constraints. The IEEE 23rd International Conference on Advanced Information Networking and Applications; 2009 May 26-29. Bradford, United Kingdom; 2009.

3. Lin G, Lazarescu M, Soh S, et al. Energy-aware two link-disjoint paths routing. IEEE International Conference on High Performance Switching \& Routing IEEE; 2013.

4. Li X, Cuthbert L. On-demand node-disjoint multipath routing in wireless ad hoc networks. IEEE International Conference on Local Computer Networks IEEE; 2004.

5. Ash A. A hybrid protocol for identification of a maximal set of node disjoint paths in mobile ad hoc networks. IEEE International Conference on Networks IEEE; 2008.

6. Nagaratna M, Srinivas PVS, Prasad VK, et al. Computation of multiple paths in MANETs using node disjoint method. International Journal of Computer Science Issues 2011.

7. Sun Z, Xie Z, Chen Z. Algorithm design for disjoint path problem. Computer Engineering 2013.

8. Chen W, Xiao W, Parhami B. An efficient construction of node disjoint paths in OTIS networks. Advanced Parallel Processing Technologies, 7th International Symposium, APPT 2007; 2007 Nov 22-23; Guangzhou, China; Proceedings DBLP; 2007.

9. Newport KT, Varshney PK. Design of survivable communications networks under performance constraints. IEEE Transactions on Reliability 1991; 40(4): 433-440.

10. Lipson HF, Mead NR, Linger RC. Requirements 
definition for survivable network systems 1998.

11. Rohrer JP, Sterbenz JPG. Predicting topology survivability using path diversity. International Congress on Ultra Modern Telecommunications \& Control Systems \& Workshops IEEE; 2011.

12. Liang Q, Modiano E. Survivability in time-varying networks. IEEE Transactions on Mobile Computing 2015; PP(99): 2668-2681.

13. Liu W, Pawlikowski K, Sirisena H. Algebraic connectivity metric for spare capacity allocation problem in survivable networks. Computer Communications 2011; 34(12): 1425-1435.

\section{Appendix: $T S_{\text {lnndp }}$ Compared to AND}

\section{TOPO1 (Node:5, Link: Increasing from 4 to 10)}
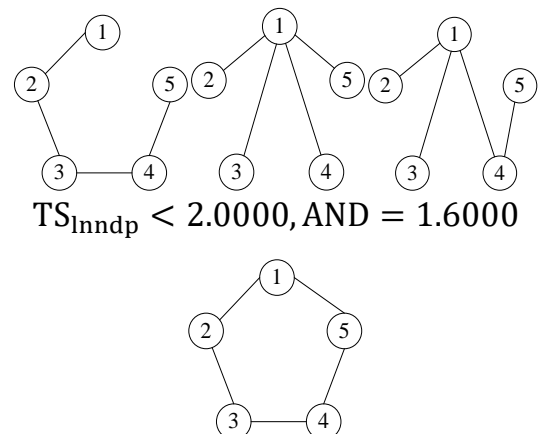

$\mathrm{TS}_{\text {lnndp }}=2.0000, \mathrm{AND}=2.0000$
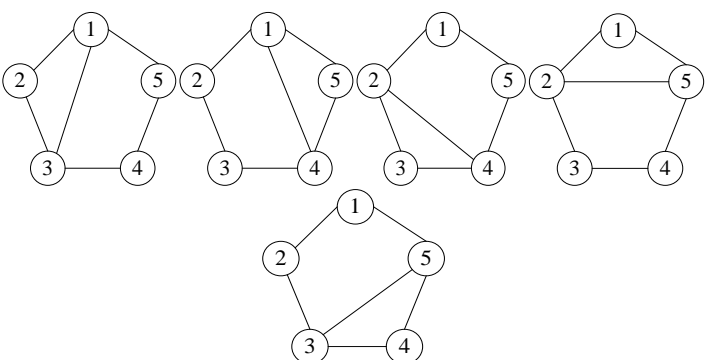

$\mathrm{TS}_{\text {lnndp }}=2.0476, \mathrm{AND}=2.4000$

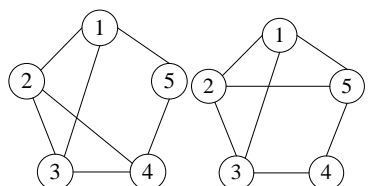

$\mathrm{TS}_{\text {lnndp }}=2.0909, \mathrm{AND}=2.8000$

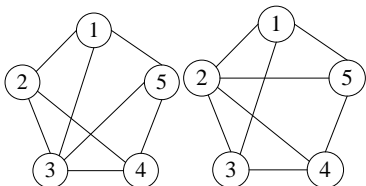

$\mathrm{TS}_{\text {lnndp }}=3.0000, \mathrm{AND}=3.2000$

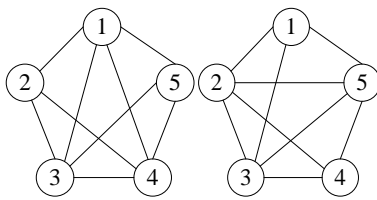

$\mathrm{TS}_{\text {lnndp }}=3.0909, \mathrm{AND}=3.6000$

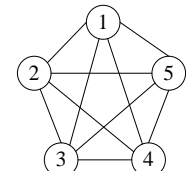

$\mathrm{TS}_{\text {lnndp }}=4.0000, \mathrm{AND}=4.0000$

TOPO2(Node:6, Link: Increasing from 7 to 15)

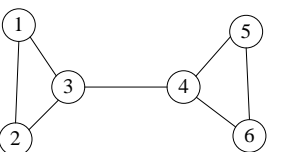

$\mathrm{TS}_{\text {lnndp }}<2.0000, \mathrm{AND}=2.3330$

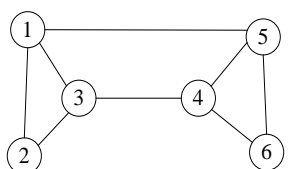

$\mathrm{TS}_{\text {lnndp }}=2.0625, \mathrm{AND}=2.6667$

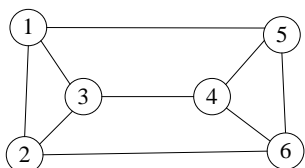

$\mathrm{TS}_{\text {lnndp }}=3.0000, \mathrm{AND}=3.0000$

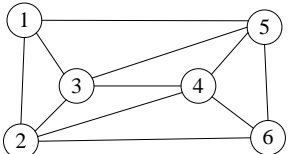

$\mathrm{TS}_{\text {lnndp }}=3.1000, \mathrm{AND}=3.6667$

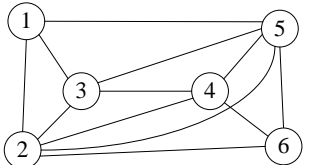

$\mathrm{TS}_{\text {lnndp }}=3.1346, \mathrm{AND}=4.0000$

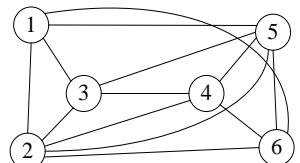

$\mathrm{TS}_{\text {lnndp }}=4.0164, \mathrm{AND}=4.3333$

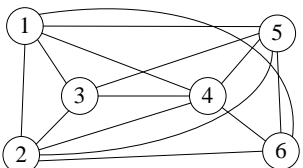

$\mathrm{TS}_{\text {lnndp }}=4.0909, \mathrm{AND}=4.6667$

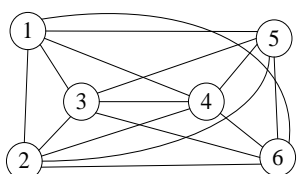

$\mathrm{TS}_{\text {lnndp }}=5.0000, \mathrm{AND}=5.0000$ 